\title{
El suicidio en disputa: aproximación crítica a la asociación entre suicidio y sexualidades no heteronormativas
}

\section{Suicide in dispute: a critical approach to the association between suicide and non-heteronormative sexualities}

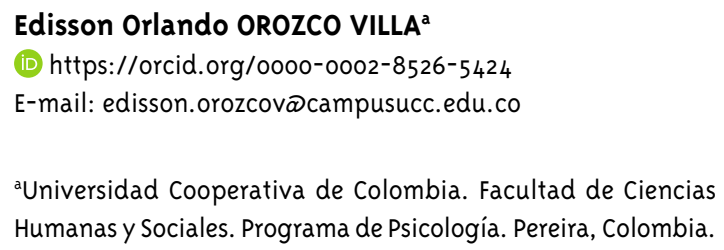

\section{Correspondência}

Antiguo complejo educativo "La Julita". Barrio Pinares, Pereira, Risaralda, Colombia.

\section{Resumen}

El presente artículo tiene como objetivo problematizar la manera en cómo los grupos sociales con sexualidades no heteronormativas han sido asociados con el suicidio y las consecuencias de esta asociación en los ámbitos académicos y sociales. La justificación de esta reflexión consiste en indicar que esta vinculación tiene implicaciones más allá de las posiciones teóricas de los investigadores, reconociendo que la asociación produce efectos sobre cómo es significada socialmente la sexualidad. Para ello, el artículo se divide en cuatro apartados. En los dos primeros apartados se analiza la manera en que la noción de riesgo epidemiológico se convierte en un rasgo identitario en las poblaciones con sexualidades no heteronormativas. En el tercer apartado se propone una reflexión sobre la investigación académica acerca de la asociación entre género y suicidio. Y en el último apartado, se expone una propuesta de interpretación del comportamiento suicida desde la perspectiva de género que problematice el sentido afectivo y político de la asociación.

Palabras clave: Suicidio; Género; LGBTIQ; Riesgo; Suicidología; Salud Mental. 
This study analyzes how social groups with nonheteronormative sexualities are often associated with suicide and posits some consequences of this association in academic and social contexts. This reflection contributes to a necessary conversation that extends beyond the theoretical positions of researchers and has a social effect. In this paper I highlight some of the experienced effects produced by the association between suicide and sexual diversity by acknowledging how sexuality assumes a specific social meaning. The article is divided into four sections. In the first two, I analyze how the notion of epidemiological risk becomes an identity trait in populations with non-heteronormative sexualities. In the third section, I reflect on academic research regarding the association between gender and suicide. The last section presents a proposal for interpreting suicidal behavior from a gender perspective that problematizes the affective and political meaning of the association.

Keywords: Suicide; Gender; LGBTIQ; Risk; Suicidology; Mental Health.
Por otra parte, no debemos subestimar la fuerza de patologización de la diagnosis, especialmente sobre gente joven que puede que no tenga los recursos críticos para resistir esta fuerza. En estos casos, la diagnosis puede ser debilitadora, si no homicida: a veces asesina el alma y a veces se convierte en un factor que contribuye al suicidio. (Butler, 2006, p. 116)

En el año 2014 el suicidio de Sergio Urrego de 16 años tuvo un efecto mediático y judicial importante en Colombia. Este caso fue uno de los 1.878 que ocurrieron ese año en el país según lo indicó el Instituto Nacional de Medicina Legal y Ciencias Forenses (2014). Sin embargo, Sergio Urrego era un joven gay y su muerte evidenció una serie de prácticas de exclusión y acoso por parte de las directivas de la institución educativa donde estudiaba y también por parte de la psicóloga de la institución -la cual asumió los cargos por discriminación en 2017-.

Pero esta muerte también develó una experiencia social más amplia acerca de las sexualidades no heteronormativas ${ }^{1}$ y el suicidio: evidenció que el suicidio no se debería limitar con exclusividad a una problemática individual de salud mental. Aquí el suicidio muestra un entrecruce de saberes sobre la vida, la muerte y la sexualidad que exceden las individualidades. Provoca que aparezcan las voces de expertos, jueces, periodistas y otros ciudadanos que revelan que el suicidio es una situación con implicaciones políticas, sociales, históricas y culturales. Además, sirve para evidenciar los modos sociales de relación con estas sexualidades.

Así, hay que considerar que las nociones teóricas producen efectos en la realidad y también en la experiencia social de las personas. Las clasificaciones no solo cumplen una función de ordenamiento de ciertos objetos, sino que también indican las posiciones y actitudes que se tienen sobre aquello que es clasificado. Los conceptos

1 En este trabajo se usa la noción de sexualidades no heteronormativas para denominar aquellas experiencias de la sexualidad, el género, el deseo, el afecto y la filiación que han sido subsumidas de manera arbitraria bajo la categoría de la homosexualidad. En este sentido, es una noción que permite destacar la multiplicidad, la historicidad y el carácter cultural de la sexualidad, mientras cuestiona la heteronormatividad entendida como matriz de poder que pretende fundamentar y regular la experiencia de la sexualidad y el género desde la heterosexualidad (Guzman; Platero, 2012). 
que se producen en la práctica científica y son considerados de manera habitual como inmunes de prejuicios, también reproducen con sofisticación las representaciones y prácticas de su contexto de producción. Por ejemplo, los procesos de racialización tuvieron un influjo sobre la práctica médica cuando sirvieron para sustentar la eugenesia racial o para justificar la deficiencia en la salubridad en algunos grupos poblacionales en Latinoamérica (Noguera, 2003). Esto muestra que las categorizaciones y clasificaciones en el campo de la salud no se alejan de sus contextos de emergencia, particularmente las asociadas a la sexualidad y el género.

Sin embargo, las consecuencias sociales de otras clasificaciones o relaciones teóricas que han sido producidas en la psicología o la psiquiatría parecen más soterradas. En este caso, se podría considerar que los efectos de la asociación del suicidio con grupos sexuales diversos no se han problematizado desde esta óptica (Jaworski, 2016). Cuando se señala que estos grupos presentan una mayor prevalencia de ideación o intento de suicidio, pocas veces se debate sobre las implicaciones sociales y políticas de esa vinculación. Es por ese motivo que resulta significativo proponer un debate que problematice los efectos epistemológicos y metodológicos que conlleva la asociación entre diversidad sexual y suicidio, pero también sobre sus derivaciones en la interpretación cultural -como en los medios de comunicación- y las políticas ligadas al suicidio. ${ }^{2}$

\section{Suicidio y diversidad de género: la producción social del riesgo}

Preguntarse sobre qué es un suicida puede resultar en apariencia algo obvio: se diría que es una persona que ha elegido finalizar con su vida. Pero más allá de esta aparente obviedad, la definición sobre el suicidio y el suicida cobra relevancia debido a las implicaciones metodológicas y la complejidad del estudio del comportamiento suicida. La Organización Mundial de la Salud (OMS, 2014) señala que anualmente ocurren más de 800.000 casos de suicidios en el mundo. Una cifra que sirve para indicar la magnitud del fenómeno, pero que asimismo homogeniza y generaliza a las personas que mueren. Por esta razón, las preguntas sobre las características sociales y culturales, que son mucho más singulares y difíciles de estimar por la epidemiología, son centrales para comprender cómo se produce socialmente la noción de suicida (Marsh, 2015).

Inicialmente, una de las formas de producir una definición del suicida parte de la identificación de poblaciones con mayor propensión al suicidio. En la actualidad, los grupos de riesgo son múltiples y disímiles entre ellos (Overholser; Braden; Dieter, 2012). Algunos estudios señalan que las personas con problemas mentales, los consumidores de alcohol, los reclusos, los refugiados, los veteranos de guerra y los migrantes tienen una mayor probabilidad de suicidarse (OMS, 2014). Estos grupos se identifican y codifican bajo criterios estadísticos que permiten entender generalidades del comportamiento suicida en números amplios de la población. Pero pasa desapercibido que las cifras también sirven para producir representaciones y significados sobre el suicidio y el suicida. Los datos cuantitativos producen explicaciones sobre el fenómeno y, al mismo tiempo, configuran las tendencias en la investigación científica e inciden en las agendas políticas de la salud que codifican e intervienen sobre determinados grupos poblacionales que se tipifican bajo el riesgo (White, 2015).

Vinculado con lo anterior, un primer aspecto que sobresale en la producción social del suicida es el desplazamiento de los datos epidemiológicos a contextos y grupos sociales más amplios. Este ejercicio de traducción de los datos epidemiológicos a los medios de comunicación trae consecuencias que pocas veces son reconocidas y previstas por los expertos. La labor de los investigadores se centra, casi siempre, en la producción de los datos, su sistematización y posterior análisis; mientras la reflexión sobre los efectos de la investigación

\footnotetext{
2 Para el desarrollo del presente artículo se analizaron fuentes bibliográficas en español, portugués e inglés que abordan la asociación entre suicidio y sexualidades no heteronormativas. La búsqueda de los artículos se realizó por medio de combinación sistemática de palabras clave en las bases de datos (Scopus, PubMed, SciELO, Dialnet y Redalyc). Para el desarrollo de la reflexión crítica fueron centrales algunas revisiones sistemáticas de literatura especializada, las cuales aparecen referenciadas en el artículo.
} 
académica sobre el suicidio en la sociedad no parece una prioridad. La anterior situación se hace evidente cuando los medios de comunicación utilizan la información experta sobre el suicidio y, más allá de establecer si sus usos son "correctos" o "incorrectos", se evidencian efectos que son más notorios y con mayor alcance que aquellos que produciría, incluso, la bibliografía especializada (Johnson, 2016).

Si se considera lo anterior, resulta poco fructífero centrarse en emitir juicios de valor sobre los medios de comunicación o las significaciones del suicidio en la cultura popular, sin examinar el papel del discurso experto. Por el contrario, la investigación sobre el suicidio podría preguntarse, de manera contundente, por los efectos que tienen las construcciones académicas sobre las interpretaciones sociales y culturales del fenómeno. Por ejemplo, el análisis de contenido de información periodística sobre el suicidio ha resultado útil para conocer cómo los medios representan al suicida, pero pocas veces se proponen análisis de los prejuicios sociales, políticos y culturales asociados al suicida en los medios de comunicación (De Pinho; Kantorski; Hernández, 2009). Esta tarea implicaría proponer matrices conceptuales críticas e interpretativas del análisis del discurso que van más allá de los análisis estadísticos (Orozco Villa, 2019). Algo similar ocurriría si se propusiera un análisis de los efectos del saber experto en la vida cultural.

Ahora bien, considerando las implicaciones anteriores, en varias investigaciones se han planteado que la ideación suicida y los intentos de suicidio son mayores en grupos con sexualidades no heteronormativas que en los heterosexuales (Flowers, 2001; King et al., 2008; Meyer; Dietrich; Schwartz, 2008; Stone et al., 2014). Estos estudios tienden a relacionar variables vinculadas con la homofobia, la discriminación y el soporte social con respuestas individuales asociadas con el suicidio. Muchas de estas investigaciones reconocen las dificultades que existen para establecer relaciones de causalidad entre las variables estudiadas; pero, más allá de las advertencias teóricas y metodológicas de los expertos, en las traducciones sociales se suele confundir la asociación de variables con relaciones de causalidad. Por esta razón, es habitual observar cómo en muchos medios de comunicación se expresa que la sexualidad diversa sería una causa del comportamiento suicida. Premisa que puede ser naturalizada por la sociedad y también en los ámbitos académicos, deviniendo una posible patologización de las sexualidades no heteronormativas (McDermott; Roen; Scourfield, 2008).

La naturalización hace que los fenómenos sociales y psicológicos pierdan su complejidad, provocando que muchos de los análisis e intervenciones redunden en lecturas repetitivas o intervenciones burocratizadas del suicidio. En consecuencia, el costo de la asociación entre sexualidades no heteronormativas y suicidio también es político debido a que, más allá de las lecturas informadas de los expertos, se producen narrativas que tienen efectos sociales y se sustentan en criterios científicos.

Un ejemplo de lo anterior es el análisis de Waidzunas (2012) acerca la asociación epidemiológica entre la categoría "juventud gay” y el riesgo suicida. El autor señala que el interés por el estudio de la relación del suicidio con la categoría de "juventud gay” inicia en los años ochenta en los Estados Unidos. Esta tendencia está marcada por la incidencia política de los activistas gais en los ámbitos públicos y sus demandas de inclusión en los sistemas de salud. Sin embargo, Waidzunas muestra que, a partir del informe gubernamental realizado por Paul Gibson en 1989, se inicia una paulatina identificación de los gais como una población con mayor riesgo de intento de suicidio. Así un grupo de personas que antes se encontraba marcado por violencias médicas, políticas e intelectuales, se convierte también en un nuevo grupo de riesgo asociado con el suicidio.

La manera en que Waidzunas (2012) analiza la asociación entre estas categorías parte de la noción propuesta por Bruno Latour denominada "black boxing”. Esta noción permite comprender cómo ciertos enunciados que tienen validez científica surgen de las afirmaciones realizadas por expertos y son estimadas como válidas sin analizar sus contextos de emergencia teórica o empírica. En este caso, Waidzunas (2012) muestra que la asociación entre homosexualidad, adolescencia y suicidio es construida por instituciones políticas, científicas y sociales. De esta manera, la constante repetición de los datos, la realización de eventos que debaten sobre el suicidio en personas con sexualidades no 
heteronormativas, las intervenciones de los expertos y otros actos de visibilidad, tanto académica como mediática, legitiman una asociación que es asumida como un hecho evidente, aunque muchas veces los datos no sean concluyentes o no expresan relaciones de causalidad.

Si bien es posible reconocer que existen datos empíricos que podrían respaldar la asociación entre sexualidades no heteronormativas con comportamientos suicidas, la noción de riesgo se produce bajo criterios epidemiológicos que se centran en algunas características biológicas, psicológicas y sociales. Se entiende que el objetivo de conocer estos factores de riesgo consiste en encontrar bases empíricas que respaldan el diseño de estrategias de intervención que sean focalizadas y adecuadas; pero de manera simultánea ocurren otros efectos que no son evidentes cuando se asume que un grupo poblacional se encuentra en riesgo. Esto significa que el riesgo, siendo una categoría de la epidemiología con delimitaciones epistemológicas precisas (Almeida Filho; Castiel; Ayres, 2009), adquiere una carga semántica que sirve para producir un nuevo modo de estigmatización social. En este caso, es una asociación que puede servir para legitimar un significado sobre la sexualidad no heteronormativa marcado por la incidencia del suicidio.

Por este motivo, cuando los datos epidemiológicos se convierten en un rasgo identitario, los grupos que han sido objeto de análisis deben afrontar nuevas problemáticas. Por ejemplo, cuando se analiza la manera en que históricamente se produjo una relación entre el VIH y la homosexualidad, se encuentra que no solo tuvo efectos técnicos y salvó vidas, sino que también produce una serie de identidades marcadas por la enfermedad. Este aspecto lo expuso Sontag (2003) cuando señalaba que estar infectado con VIH en los años ochenta era sinónimo de ser reconocido como parte de un colectivo de riesgo o de una "comunidad de parias". Un grupo que evocaba inmediatamente a varones homosexuales que padecían "la enfermedad rosa".
En la actualidad, la situación no es muy diferentey, en este caso particular, el suicidio se instaura paulatinamente como otro rasgo identitario de un grupo poblacional. Se asume que la muerte por mano propia es una situación que pareciera inherente a la experiencia de vida de las personas con sexualidades no heteronormativas, lo cual puede redundar en nuevas formas de estigmatización y de invisibilización de situaciones políticas que históricamente han oprimido a estos grupos sociales.

La noción de riesgo es más que una categoría epidemiológica o clínica, pues opera también como una representación cultural que dinamiza y ordena las relaciones sociales con las poblaciones identificadas. En este sentido, los discursos expertos proponen una lectura del suicidio que, al igual que ocurrió con el VIH, se convierte poco a poco en un rasgo identitario de un grupo. Este punto puede resultar controversial debido a que el discurso experto propone un uso preciso y riguroso de los conceptos que supone una neutralidad valorativa; pero en algunas ocasiones, se desconoce que las enfermedades también reproducen expresiones simbólicas que hacen parte de un sistema de valores más amplios que la propia práctica científica. ${ }^{3}$

Las gramáticas de los expertos sirven para construir, siempre de maneras variadas, algunas interpretaciones que son centrales sobre los fenómenos sociales que son estudiados. Se debería reconocer que esta situación no es responsabilidad directa de los investigadores; pero las traducciones que se hacen socialmente son siempre complejas y mediadas por la carga histórica de los receptores de la información (por ejemplo, grupos con capacidad de decisión política, pero sin conocimiento técnico de los temas). Así el reconocimiento de los efectos políticos y sociales de la producción de saber es una responsabilidad ineludible en la actualidad y mucho más si vincula grupos sociales que tradicionalmente han sido estigmatizados.

Por las razones anteriores, cuando se propone una problematización de la noción de riesgo suicida en las sexualidades no heteronormativas, resulta

\footnotetext{
3 No obstante, se debe reconocer que también, bajo ciertas circunstancias políticas, estos rasgos pueden ser pensados como identidades estratégicas (Spivak, 2003).
} 
importante pensar en una política de la traducción. Esto significa reconocer que los análisis científicos más rigurosos siempre pueden ser traducidos, incluso en los mismos ámbitos académicos, como marcas de identidad sobre aquellos grupos o individuos que son estudiados. En ese sentido, no son suficientes las reflexiones epistemológicas y metodológicas, también son centrales las reflexiones éticas y políticas sobre el sentido de la producción de conceptos sobre el suicidio en sexualidades no heteronormativas. De igual manera, es central reconocer que la noción de riesgo, que hace parte de una tradición epidemiológica hegemónica, se puede convertir con facilidad en una forma subrepticia de estigmatización de estas sexualidades en los mismos contextos académicos.

\section{Contenido del riesgo: determinismo biológico y despolitización del suicidio}

Hasta este punto se ha discutido cómo por medio de la noción de riesgo es posible configurar rasgos de identidad, pero también es importante problematizar el contenido del riesgo. En otras palabras, para comprender cómo se produce el suicida no es suficiente con saber quién se suicida (población de riesgo), es central identificar cuáles son los elementos asociados al comportamiento (factores de riesgo). En este sentido, las investigaciones sobre suicidio señalan en la mayoría de los casos que es un fenómeno multicausal (OMS, 2014). Esto significa que el suicidio puede estar mediado por aspectos que van desde características biológicas y psicopatológicas, pasando por estilos cognitivos de procesamiento de la información, hasta la incidencia de dinámicas familiares y situaciones económicas adversas (Ellis, 2008; Guibert Reyes; Torres Miranda, 2001; Nordt et al., 2015; Sudol; Mann, 2017). Todos estos elementos tan heterogéneos configuran representaciones sobre el suicidio y el suicida, los cuales muestran una multiplicidad del fenómeno que puede redundar en descontextualización.

En el caso de las personas con sexualidades no heteronormativas, se han establecido algunos tipos diferenciados de riesgo. Se ha señalado una asociación con una prevalencia mayor de trastornos mentales, consumo de sustancias psicoactivas y alcohol. También se ha planteado que la homofobia (percibida, experimentada e internalizada) y el abuso sexual o psicológico inciden en la manifestación de ideaciones y comportamiento suicida. En consecuencia, los discursos de la medicina, la psiquiatría y la psicología se basan, la mayoría de las veces, en datos epidemiológicos para enunciar una serie de situaciones y contextos que parecen ser claves en el momento del estudio del suicidio en este grupo poblacional.

De esta manera, estas lecturas sobre los riesgos del suicidio en estos grupos han sido útiles para reflexionar sobre el fenómeno y orientar prácticas de intervención, pero causan algunos inconvenientes analíticos que no suelen ser discutidos. Un primer inconveniente tiene que ver con la sobredeterminación biológica de la experiencia social. Este proceso hace que se centre la atención, casi de manera exclusiva, en lecturas biologicistas que procuran la medicalización del comportamiento suicida centradas en la individualidad. La aclaración que plantea Pineda (2013, p. 229) es pertinente para entender esta cuestión:

No se desconoce el elemento biológico implicado en el suicidio, ni las enfermedades mentales que lo precipitan, como es el caso de la depresión, que ha jugado un importante papel en la predicción de la conducta suicida. No obstante, la mayor proporción de ideación e intento de suicidio en minorías sexuales no da lugar para otorgarles el calificativo de suicidas a personas lesbianas, gay y bisexuales.

Si bien, es importante la explicación de los procesos psicobiológicos que pueden incidir en el comportamiento suicida, estos pueden quedar limitados cuando son situados fuera de contexto. Hasta el momento, los estudios no han logrado ser contundentes en evidenciar la incidencia directa de factores biológicos sobre el comportamiento suicida más allá de su ocurrencia asociada con trastornos mentales. A pesar de esto, esa interpretación tiende a ser normalizada en los marcos teóricos de la explicación del suicidio, y se considera que el comportamiento suicida se puede explicar desde características biológicas. Por esta razón, se concibe el comportamiento suicida más como efecto de una reacción biológica que propiamente 
un comportamiento estructurado con un sentido cultural, social, político y subjetivo.

En algunas investigaciones aparecen referencias a la homofobia o el estigma social como factores de riesgo asociados al comportamiento suicida (Ferlatte et al., 2017; Rimes et al., 2018). Se señala que se pueden considerar estas situaciones como desencadenantes de trastornos de los estados de ánimo o enfermedades mentales. Esta interpretación puede resultar limitada y hace que se asuma la homofobia ${ }^{4}$ o el estigma social como situaciones sociales que ocurren de manera homogénea en todos los contextos, omitiendo las singularidades históricas y culturales. Son interpretaciones que privilegian rasgos individuales, pero que, paradójicamente, se distancian de la experiencia subjetiva de la vivencia del suicidio.

En este sentido, algunos modelos teóricos, como el estrés de las minorías (Meyer; Dietrich; Schwartz, 2008), ofrecen importantes herramientas para la explicación del comportamiento suicida en personas con sexualidades no heteronormativas; pero el uso investigativo del modelo sigue concibiendo el comportamiento suicida como producto de formas de procesamiento y afrontamiento que privilegian lo individual. Además, las lógicas de investigación se centran en ofrecer mediciones sin plantear explicaciones de los contextos en los que ocurre el fenómeno. Incluso, el uso de la adjetivación minoría resulta problemático debido a que sigue sosteniendo una lectura que establece interpretaciones hegemónicas sobre la sexualidad y su asociación con el cálculo estadístico. Seguir pensando la sexualidad en relación con las nociones de minorías o mayorías puede convertirse en una limitación que es producto del desconocimiento de las interpelaciones teóricas y políticas que se han hecho sobre la sexualidad y el género en los últimos años.

Los modelos que son usados habitualmente para entender el fenómeno del suicidio otorgan una primacía a componentes biológicos (respuestas distresantes) y hacen que exista una sobredeterminación de los rasgos individuales; mientras las configuraciones sociales quedan ubicadas como parte de cierta "escenografía". Así la definición de los "factores de riesgo psicosocial” que figuran constantemente en los modelos ecológicos o biopsicosociales, a causa de su configuración teórica, limitan la comprensión de prácticas complejas de significación cultural y construcción histórica de la experiencia del morir. Las limitaciones analíticas de estas propuestas han movilizado el desarrollo de alternativas teóricas que entienden el comportamiento suicida, incluida su relación con el género y la sexualidad, desde miradas que involucran interpretaciones minuciosas de los discursos sociales y culturales en donde se produce el suicidio, considerando que el morir por mano propia es una práctica compuesta por una historicidad que no se limita de manera exclusiva a los datos cuantitativos (White, 2015).

Tanto la sobredeterminación de los aspectos biológicos como la saturación de una identidad suicida asociada a las sexualidades no heteronormativas ilustran dos maneras de interpretar la asociación entre categorías y la producción del riesgo. A pesar de ello, no hay que descuidar que existen otras reiteraciones argumentativas y conceptuales en los estudios sobre el suicidio y la asociación con estas sexualidades. Así, por ejemplo, la discriminación es un tema constante de discusión en las investigaciones, convirtiéndose en una categoría o variable que es referida como elemento causal en el comportamiento suicida. De esta manera, encontramos nuevamente una homogeneización y la generalización de algunas situaciones sociales. Igualar, por ejemplo, la discriminación religiosa con la discriminación racial o de género -como ocurre con el uso del modelo de estrés de las minoríasindica una aproximación parcial del fenómeno. La discriminación interpretada como una generalidad es una categoría analítica limitada debido a que niega la variabilidad social del fenómeno, también invisibiliza construcciones ideológicas y componentes políticos que precarizan la vida.

4 Es necesario aclarar que la noción de homofobia es pensada como un dispositivo productor de violencia simbólica o física que se despliega por medio de la discriminación, la exclusión o la destrucción de los cuerpos de las personas con sexualidades no heteronormativas. Cada expresión de homofobia debe ser entendida desde la singularidad, comprendiendo que su despliegue es relativo a las características del grupo o las personas que afecta. 
En este contexto, es importante considerar que las discriminaciones -en plural- son prácticas que se producen y diferencian bajo marcos históricos, políticos y culturales específicos. Asumir esta cuestión permite que no se sustraiga la complejidad y la singularidad del fenómeno del suicidio. Así, el anterior argumento no consiste en negar la particularidad de las experiencias de discriminación, por el contrario, procura comprender cómo cierto tipo de prácticas se instauran socialmente como discriminatorias, y las interpretaciones subjetivas hacen que el suicidio se convierta en parte del repertorio social para posicionarse frente a esta práctica -lo cual estaría lejos de entender el suicidio como una llana respuesta-. Esto hace que se cuestionen lecturas que sostienen visiones hegemónicas sobre el género y la sexualidad en el ámbito de la investigación en temas de salud mental (Fish, 2008).

No hay que desconocer que algunas prácticas políticas tienen incidencia en la salud mental de las personas con sexualidad no heteronormativa e incluso pueden tener efectos sobre los comportamientos suicidas. La imposibilidad para acceder a los derechos asociados con el matrimonio o la adopción puede tener costos psicológicos y sociales muy altos. En la actualidad, en muchos países latinoamericanos se observa que amplios sectores políticos son reticentes en mantener o ampliar los derechos de los grupos con sexualidad diversa. Estas formas de discriminación y estigmatización son producto de políticas sistemáticas y no de comportamientos sociales espontáneos. En ese sentido, en cada contexto existen instituciones y agentes con una historicidad específica que promueven la discriminación. Por esta razón, pensar en una forma única y general de discriminación reduce la potencia política de los análisis y también las formas de intervención.

\section{¿Homofobia internalizada o internalización de la homofobia en la investigación?}

Si bien han existido transformaciones en las maneras en que se conceptualizan las sexualidades no heteronormativas en la psicopatología (Drescher, 2015), en ciertos ámbitos persisten estigmas que siguen consolidando formas de discriminación. Recientemente se ha observado que la producción académica vinculada con el estudio de la diversidad sexual ha estado atravesada por posiciones críticas que cuestionan algunas posibles derivas homofóbicas en el campo académico. No obstante, De la Espriella (2007) muestra que algunas perspectivas teóricas de la psiquiatría se ubican en posiciones que legitiman y sostienen la patologización de las sexualidades no heteronormativas de manera implícita. Se debe señalar que estas formas de homofobia no se reducen al ámbito conceptual, también trascienden a los significados sobre la diversidad sexual en los profesionales de la salud y en la oportunidad en la atención de las personas.

Esta cuestión tiene implicaciones significativas pues señala múltiples barreras en el acceso a la salud. Un ejemplo se encuentra en la investigación realizada por Ritterbusch, Correa Salazar y Correa (2018) con un grupo de mujeres transexuales en Colombia, en la cual se evidenciaron formas sistemáticas de violencia en algunos ámbitos de atención médica. Si bien la legislación colombiana no plantea diferencias en la atención, en las narrativas de las participantes se evidencian múltiples formas de discriminación y maltrato sistemático. En este caso, las autoras proponen que estas discriminaciones se encuentran articuladas con la formación académica y el entrenamiento del personal de salud; aspectos que deberían incluso producir reformas curriculares que sean sensibles con la diversidad sexual y de género. Estos casos sirven para proponer una reflexión que evidencie la interseccionalidad de las discriminaciones, en las cuales se muestra confluencia entre situaciones políticas e históricas del contexto, las prácticas académicas del discurso médico y las posiciones subjetivas del personal de atención (Fish, 2008).

El ejemplo anterior puede resultar útil para pensar que la relación entre el saber experto y la homofobia tiene consecuencias concretas en la vida de las personas, pero también se evidencia cómo los contextos educativos en los cuales se forman las personas encargadas de la salud de la población reproducen estas prácticas. En ese mismo sentido, es central analizar cómo las actitudes de los profesionales de la salud pueden incidir sobre 
la oportunidad y la calidad en la atención. Las barreras en la atención en la salud ilustran formas de discriminación estructural que acentúan las brechas sociales entre grupos poblacionales. En este caso, se puede considerar que las barreras en la atención no solo son producto de situaciones institucionales, sino que también están en juego las actitudes del personal de salud.

Si consideramos que las actitudes de los profesionales de la salud inciden de manera negativa sobre la atención, este asunto es relevante cuando se piensa desde la óptica de la atención y prevención del suicidio. Por esta razón, cuando se niega la existencia de prenociones acerca de las sexualidades no heteronormativas, es posible que se limite el desarrollo de los enfoques diferenciales de atención en salud y se reproduzcan prácticas marcadas por la homofobia. En este sentido, como proponen Nieto y Orozco (2016), la investigación de las actitudes sobre el suicidio en los profesionales de la salud es clave para el desarrollo de estrategias de atención y prevención del comportamiento suicida.

En este marco de discusión se evidencia cómo se reproducen formas de estigmatización en los contextos encargados de la atención en salud. Esta cuestión se complejiza cuando se piensa que ocurre algo similar en los ámbitos investigativos. Si bien los trabajos de Jaworski (2016) y Waidzunas (2012) proponen una crítica sobre la asociación conceptual entre suicidio y diversidad sexual, se evidencia que la reflexión sobre la internalización de la homofobia -y cualquier rechazo a las sexualidades no heteronormativas- puede pasar desapercibida en el ámbito de la investigación. Hay que considerar que los investigadores pueden basar sus planteamientos sobre prejuicios sociales y culturales indelebles, para que sus métodos de investigación sean rigurosos y sustentados epistemológicamente.

Por ejemplo, Kitts (2005) propone que en algunos campos de conocimiento los temas vinculados con el género han sido estudiados de manera amplia, pero señala que existe una limitación en la investigación en la medicina y la psicología. La hipótesis del autor se centra en que, al existir una posible asociación entre el tema estudiado con la orientación sexual de los investigadores, puede ocurrir que se evite trabajar en estos temas por temor a ser identificado como parte de un grupo con sexualidad no heteronormativa. Si bien es una hipótesis que podría ser problematizada, sirve para situar las implicaciones extrateóricas que producen los estudios de género en campos del saber que se caracterizan por relaciones sociales heteronormativas y que en su desarrollo histórico patologizaron y medicalizaron las sexualidades que no suponían normales. ${ }^{5}$ Esto se afirma cuando consideramos que los estudios de género han sido objeto de sospecha tanto intelectual como política en muchos contextos académicos y, en especial, en Latinoamérica. Al respecto, la noción de injusticia hermenéutica desarrollada por Pérez (2019) puede resultar esclarecedora para evidenciar algunas dificultades de los estudios de salud mental para comprender la singularidad del comportamiento suicida en esta población:

Esto -la injusticia hermenéutica- puede darse mediante la inexistencia de categorías hermenéuticas adecuadas, un rechazo directo de las nociones que desarrollan las comunidades, una falta de voluntad para comprenderlas o incorporarlas, y/o la ilusión de que pueden interpretar su propia realidad de manera satisfactoria a partir de las categorías ofrecidas por los grupos que lxs marginan, lo que en otro trabajo hemos llamado “espejismo hermenéutico”. (Pérez, 2019, p. 9o)

Igualmente, el estudio de la asociación teórica del suicidio con las sexualidades no heteronormativas se caracteriza por la producción investigativa que se publica mayoritariamente en inglés y fuera

\footnotetext{
5 Kitts (2005, p. 623, la traducción es mía) expresa esta situación de la siguiente manera: "No me sorprendería si los lectores de este artículo asumieran que yo fuera gay. Este era un riesgo que dudaba en tomar. Desafortunadamente, es un riesgo que algunos médicos no estarían dispuestos a tomar por miedo a comprometer sus carreras. Este miedo impide que los temas homosexuales importantes sean escuchados en la corriente dominante. Goldfried declaró que, a pesar de la creciente literatura sobre temas homosexuales, la psicología convencional ha tendido a ignorar gran parte del trabajo que se ha realizado en esta área. Por lo tanto, cuestiones importantes, como el suicidio entre los adolescentes homosexuales, permanecen invisibles no solo para la psicología convencional, sino también para la atención médica general.”
} 
de los contextos latinoamericanos (Pineda, 2013; Teixeira-Filho; Rondini, 2012). Tal como lo expresan Tomicic et al. (2016) en una revisión sistemática de la cuestión, la mayoría de los estudios se producen en países anglosajones, evidenciando situaciones propias de las geopolíticas del conocimiento. Por esta razón, pensar un análisis del suicidio desde el enfoque de género en Latinoamérica es central. En este punto, no pueden pasar desapercibidas las posturas ideológicas que inciden cada vez más en el ámbito político y que limitan las agendas investigativas asociadas a los estudios de género. Esto mostraría cómo los investigadores se encuentran bajo relaciones sociales y significaciones culturales que los atraviesan y que pueden incidir en sus perspectivas teóricas. En este caso, se podría invisibilizar un número amplio de situaciones de violencia -entre ellas el suicidio- en personas con sexualidades no heteronormativas.

En definitiva, se puede observar cómo la investigación sobre el suicidio en estos grupos se encuentra marcada por intereses extrateóricos. Esto significa que más allá de las precauciones teóricas y metodológicas que proponen los investigadores en el momento de diseñar sus investigaciones, existe una serie de fenómenos sociales y políticos implícitos que inciden en esta clase de estudios. Por esta razón, como se expresaba previamente, es importante plantear un análisis constante y riguroso de los prejuicios de género que pueden existir en la investigación y las teorías que son producidas. Quizás una manera de lograr este objetivo consista en pensar la cuestión del suicidio desde un enfoque crítico de género.

\section{¿El suicidio en disputa? Posibles aportes de los estudios de género al estudio del suicidio}

Pensar los aportes de los estudios de género a la interpretación del comportamiento suicida permite considerar algunos argumentos propuestos previamente. Inicialmente puede resultar esclarecedor cuestionar las posturas esencialistas del comportamiento suicida (Marsh, 2015). Esto significa comprender el fenómeno desde sus contextos y no como una expresión inherente a la patología, lo cual hace que se privilegie la comprensión de los aspectos locales de orden social, político y cultural en que ocurren los suicidios. Esta posición plantea cuestionamientos de los modelos e intervenciones que tienden a medicalizar este comportamiento.

Uno de los campos que ha resultado importante para lograr una comprensión desencializadora del comportamiento suicida ha sido el movimiento de la suicidiología crítica y los estudios críticos del suicidio (White, 2015). Desde esta perspectiva, se ha considerado que el problema del suicidio se inscribe en lógicas sociales e históricas que son amplias y que se distancian de prácticas que interpretan el fenómeno desde una óptica patológica. Por este motivo, la noción de género que es adoptada por la suicidiología crítica cuestiona las lecturas biologicistas sobre el sexo y reconoce el sentido cultural de la sexualidad que se inscribe en el comportamiento suicida (Jaworski, 2016).

Cuestionar la esencialidad del suicidio permite que se problematicen algunas variables tradicionales de abordaje del fenómeno como ocurre, por ejemplo, con la noción de sexo. Muchos estudios sobre suicidio -incluso aquellos realizados por los Estados- se caracterizan por codificar a las poblaciones desde una diferenciación binaria entre lo masculino y femenino. Esto provoca que la cuestión del género quede subsumida a una diferenciación sexual dicotómica. Por este motivo, es central evaluar y conceptualizar las nociones de sexo y género que se realicen en los estudios vinculados con suicidio en la población general, pero sin perder de vista su carácter interseccional. Esto significa no omitir los elementos asociados a la clase, la raza, y la diversidad funcional que permean el sexo y el género.

Por otro lado, la interpretación del suicidio desde la perspectiva de género provoca que sea necesario considerar abordajes que no patologicen las sexualidades no heteronormativas. Por este motivo, es central que los abordajes investigativos puedan interpelar los marcos teóricos que conceptualizan estas formas de sexualidad desde el espectro de la patología. Si bien en la actualidad se reconoce desde los manuales diagnósticos que la orientación sexual o la diversidad del género no se puede clasificar de manera patológica, la patologización es persistente 
y se expresa en ámbitos que van más allá del diagnóstico. Las maneras de patologizar, como se mostraba previamente, se evidencian en el imaginario teórico de los campos de conocimiento en los cuales la diversidad de género se asocia con riesgo (De La Espriella, 2007). En consecuencia, uno de los puntos de partida para pensar la vinculación entre género, sexualidad y suicidio consiste en tomar distancia de la habituación teórica que interpreta la experiencia de morir por mano propia como efecto de una falla en la experiencia de vida de una persona y su sexualidad.

Pero posiblemente una de las implicaciones centrales de pensar el suicidio desde los estudios de género reside en una politización de la salud mental. Este punto implica asumir que la salud hace parte de un complejo entramado social que va más allá del discurso experto y que requiere una crítica constante a las visiones burocratizadas de la salud. Por esta razón, el lugar de los estudios de género consistiría en producir y activar visiones acerca de la muerte, el género y la sexualidad que disputen sentidos culturales sobre lo público. Que puedan desestabilizar las interpretaciones que desdibujan situaciones sociales y políticas acerca del suicidio, para ofrecer herramientas intelectuales que apelen a intervenciones en salud mental que van más allá del cálculo económico o las necesidades del mercado; evidenciando que situaciones como el suicidio están atravesadas por sistemas de interpretación que reproducen formas de dominación patriarcal.

Finalmente, uno de los aspectos que resultaría central es recuperar las voces y los saberes de aquellas personas que han transitado la experiencia del suicido, como los sobrevivientes, las familias y amigos. Una de las características de los estudios de género en los últimos años ha consistido en producir conocimientos que se sitúan y que emergen desde la experiencia de las personas, su sexualidad y su corporalidad. Esto ha permitido entender el valor de situar las prácticas de género en ámbitos locales, evitando que sean borradas por abstracciones teóricas. Por lo tanto, esta perspectiva permite que la muerte por mano propia no sea asumida como un efecto calculable de la diversidad sexual y que, por el contrario, se comprenda que algunos modos de vida están marcados por la muerte debido a situaciones de vulnerabilidad y precariedad; situaciones que suelen disolverse entre los discursos abstractos de los expertos y por algunos silencios cómplices de la sociedad. Discursos y silencios que han marcado la existencia de personas como Sergio Urrego.

\section{Referencias}

ALMEIDA FILHO, N.; CASTIEL, D. L.; AYRES, J. R. Riesgo: concepto básico de la epidemiología. Salud Colectiva, Lanús, v. 5, n. 3, p. 323-344, 2009.

BUTLER, J. Deshacer el género. Barcelona: Paidós Ibérica, 2006.

DE LA ESPRIELLA, R. Homofobia y psiquiatría. Revista Colombiana de Psiquiatría, Bogotá, DC, v. 36, n. 4, p. 718-735, 2007.

DE PINHO, L. B.; KANTORSKI, L. P.; HERNÁNDEZ, A. M. B. Análise crítica do discurso: novas possibilidades para a investigação científica no campo da saúde mental. Revista Latino-Americana de Enfermagem, Ribeirão Preto, v. 17, n. 1, p. 126-132, 2009.

DRESCHER, J. Out of DSM: depathologizing homosexuality. Behavioral Sciences, Basel, v. 5 , n. 4, p. 565-575, 2015.

ELLIS, T. El estudio de la cognición y suicidio: comienzos e hitos del desarrollo. In: ELLIS, T. Cognición y suicidio: teoría, investigación y terapia. México: Manual Moderno, 2008. p. 11-26.

FERLATTE, O. et al. Stigma and suicide among gay and bisexual men living with HIV. AIDS Care, Abingdon, v. 29, n. 11, p. 1346-1350, 2017.

FISH, J. Navigating queer street: researching the intersections of lesbian, gay, bisexual and trans (LGBT) identities in health research. Sociological Research Online, Thousand Oaks, v. 13, n. 1, p. 104115, 2008. Disponível em: <https://bit.ly/2Lonqkf>. Acesso em: 11 jan. 2021.

FLOWERS, P. Gay men and HIV/AIDS risk management. Health: An Interdisciplinary Journal for the Social Study of Health, Illness and Medicine, Thousand Oaks, v. 5, n. 1, p. 50-75, 2001. GUIBERT REYES, W.; TORRES MIRANDA, N. Intento suicida y funcionamiento familiar. 
Revista Cubana de Medicina General Integral, Havana, v. 17, n. 5, p. 452-46o, 2001.

GUZMAN, P.; PLATERO, R. L. Passing, enmascaramiento y estrategias identitarias: diversidades funcionales y sexualidades no-normativas. In: PLATERO, R. L. (Ed.). Intersecciones: cuerpos y sexualidades en la encrucijada. Barcelona: Bellaterra, 2012. p. 125-159.

INSTITUTO NACIONAL DE MEDICINA LEGAL Y CIENCIAS FORENSES. Forensis 2014: datos para la vida. Bogotá, DC: Imprenta Nacional, 2014.

JAWORSKI, K. The gender of suicide: knowledge production, theory and suicidology. New York: Routledge, 2016.

JOHNSON, H. J. Bisexuality, mental health, and media representation. Journal of Bisexuality, Abingdon, v. 16, n. 3, p. 378-396, 2016.

KING, M. et al. A systematic review of mental disorder, suicide, and deliberate self harm in lesbian, gay and bisexual people. BMC Psychiatry, Fort Lauderdale, v. 8, n. 1, art. 70, 2008.

KITTS, R. L. Gay adolescents and suicide: understanding the association. Adolescence, Amsterdam, v. 40, n. 159, p. 621-628, 2005.

MARSH, I. Critiquing contemporary suicidology. In: WHITE, J. (Ed.). Critical suicidology: transforming suicide research and prevention for 21st century. Vancouver: UBC Press, 2015. p. 15-30.

MCDERMOTT, E.; ROEN, K.; SCOURFIELD, J.

Avoiding shame: young LGBT people, homophobia and self-destructive behaviours. Culture, Health \& Sexuality, Abingdon, v. 10, n. 8, p. 815-829, 2008.

MEYER, I. H.; DIETRICH, J.; SCHWARTZ, S. Lifetime prevalence of mental disorders and suicide attempts in diverse lesbian, gay, and bisexual populations. American Journal of Public Health, Washington, DC, v. 98, n. 6, p. 1004-1006, 2008.

NIETO, L.; OROZCO, E. Reflexiones sobre la aproximación metodológica al estudio de las actitudes de los profesionales frente al suicidio. Textos y Sentidos, Pereira, n. 13, p. 181-197, 2016.
NOGUERA, C. E. Medicina y política: discurso médico y prácticas higiénicas durante la primera mitad del siglo 20 en Colombia. Medellín: Universidad Eafit, 2003.

NORDT, C. et al. Modelling suicide and unemployment: a longitudinal analysis covering 63 countries, 2000-11. The Lancet Psychiatry, Amsterdam, v. 2, n. 3, p. 239-245, 2015.

OMS - ORGANIZACIÓN MUNDIAL DE LA SALUD.

Previniendo el suicidio: un imperativo global. Washington, DC: World Health Organization, 2014.

OROZCO VILLA, E. O. Análisis crítico del discurso y suicidio: más allá de la narrativa de la enfermedad. Arbor, Madrid, v. 195, n. 794, art. a533, 2019.

OVERHOLSER, J. C.; BRADEN, A.; DIETER, L. Understanding suicide risk: identification of highrisk groups during high-risk times. Journal of Clinical Psychology, Hoboken, v. 68, n. 3, p. 349-361, 2012.

PÉREZ, M. Violencia epistémica: reflexiones entre lo invisible y lo ignorable. Revista de Estudios y Políticas de Género, Buenos Aires, v. 1, n. 1, p. 81-98, 2019.

PINEDA, C. A. Etiología social del riesgo de suicidio en adolescentes y jóvenes lesbianas, gay y bisexuales: una revisión. Psicogente, Barranquilla, v. 16, n. 29, p. 218-234, 2013.

RIMES, K. A. et al. Psychosocial factors associated with suicide attempts, ideation, and future risk in lesbian, gay, and bisexual youth: the youth chances study. Crisis: Journal of Crisis Intervention and Suicide Prevention, Boston, v. 40, n. 2, p. 83-92, 2018.

RITTERBUSCH, A. E.; CORREA SALAZAR, C.; CORREA, A. Stigma-related access barriers and violence against trans women in the Colombian healthcare system. Global Public Health, Abingdon, v. 13, n. 12, p. 1831-1845, 2018.

SONTAG, S. La enfermedad y sus metáforas: el Sida y sus metáforas. Buenos Aires: Aguilar, Altea, Taurus, Alfaguara, 2003.

SPIVAK, G. ¿Puede hablar el subalterno? Revista Colombiana de Antropología, Bogotá, DC, v. 39, p. 297-364, 2003. 
STONE, D. M. et al. Sexual orientation and suicide ideation, plans, attempts, and medically serious attempts: evidence from local youth risk behavior surveys, 2001-2009. American Journal of Public Health, Washington, DC, v. 104, n. 2, p. 262-271, 2014.

SUDOL, K.; MANN, J. J. Biomarkers of suicide attempt behavior: towards a biological model of risk. Current Psychiatry Reports, New York, v. 19, n. 6, art. 31, 2017.

TEIXEIRA-FILHO, F. S.; RONDINI, C. A. Ideações e tentativas de suicídio em adolescentes com práticas sexuais hetero e homoeróticas. Saúde e Sociedade, São Paulo, v. 21, n. 3, p. 651-667, 2012.
TOMICIC, A. et al. Suicidio en poblaciones lesbiana, gay, bisexual y trans: revisión sistemática de una década de investigación (20042014). Revista Médica de Chile, Santiago, v. 144, n. 6, p. 723-733, 2016.

WAIDZUNAS, T. Young, gay, and suicidal: dynamic nominalism and the process of defining a social problem with statistics. Science, Technology, \& Human Values, Thousand Oaks, v. 37, n. 2, p. 199-225, 2012.

WHITE, J. Shaking up suicidology. Social Epistemology Review and Reply Collective, Victoria, v. 4, n. 6, p. 1-4, 2015. 


\section{Errata}

En el artículo "El suicidio en disputa: aproximación crítica a la asociación entre suicidio y sexualidades no heteronormativas", doi 10.1590/So104-12902021200073, publicado en el volumen 31, n. ${ }^{0} 1$ de 2021 en la revista Saúde e Sociedade, en la primera página:

Onde se lia:

Edisson Orlando Orozco Villa

Leia-se:

Edisson Orlando OROZCO VILLA 